\title{
A strategy to reduce illicit drug use is effective in elite Australian football
}

\author{
Peter R Harcourt, ${ }^{1}$ Harry Unglik, ${ }^{2}$ Jill L Cook ${ }^{3}$
}

${ }^{1}$ Victorian Institute of Sport, Melbourne, Victoria, Australia ${ }^{2}$ Melbourne Sports Medicine Centre, Melbourne, Victoria, Australia

${ }^{3}$ Medicine, Nursing and Health Sciences, School of Primary Health Care, Monash University, Melbourne, Victoria, Australia

\section{Correspondence to}

Dr Jill L Cook, Medicine, Nursing and Health Sciences, Monash University, School of Primary Health Care, PO Box 527, Frankston, VIC 3199, Australia; jill.cook@monash.edu

Received 7 May 2012 Accepted 19 July 2012

\begin{abstract}
Background The World Anti-Doping Agency (WADA) prescribes that drug testing is conducted in sports competitions to detect drug use in athletes. This testing includes performance-enhancing drugs as well as illicit substances such as marijuana, amphetamines and cocaine. Illicit drugs are tested for on match days but not on non-match days. Some athletes are known to use illicit substances for recreational purposes, away from competition times and this poses a serious health and welfare issue not addressed by the usual sport drug testing regimes. This paper reports the results of the first 7 years of an illicit drug-testing programme that included non-match day testing in the elite Australian Football competition, the Australian Football League (AFL).

Methods Players in the AFL were tested for illicit drugs both in-competition and out-of-competition. Players were selected for illicit substance tests either randomly or targeted based on previous test history or time since previous test. The number of tests conducted was increased each year from 2005 to 2011 and testing was focused on high-risk times during non-competition periods.
\end{abstract}

Results There were no positive match day tests. There was a significant reduction in positive tests (19-6) for illicit drugs during non-competition periods over the 7 years $(p<0.0001)$. The reduction in positive tests may be related to player education, the greater number of tests conducted and the harm minimisation approach of the illicit drug policy.

Conclusions An illicit drugs programme using a harm minimisation strategy can work effectively alongside a sport's WADA compliant Anti-Doping Code.

\section{INTRODUCTION}

Illicit drug use for recreational purposes is a substantial issue within Western society and causes significant health issues. ${ }^{1}$ In Australian society the incidence of use of any illicit drug at least once in a lifetime has been reported to be $38 \%$ and even higher for the 20-29 age group. ${ }^{2}$ Over a 12-month period (in 2006) $11 \%$ of the population were reported to use cannabis, and $9 \%$ used amphetamines. ${ }^{2}$

The incidence of illicit substance use in elite sporting groups, based on self-report surveys, is lower than the community. Dunn et al $l^{3}$ reported that $7 \%$ of Australian athletes had used illicit drugs in the past year and elite Australian athletes had a $21 \%$ lifetime cannabis use, $9.5 \%$ for ecstasy and $6.7 \%$ for cocaine. These findings were similar to data from the USA; Green et al ${ }^{4}$ noted a selfreport incidence of recreational drug use in National Collegiate Athletic Association (NCAA) college athletes at $28.4 \%$ for marijuana, $3.1 \%$ for amphetamine and $1.5 \%$ cocaine.
Testing individuals for drug use is uncommon in the community but practiced regularly in elite sport to detect drug use. The first stand against drug use in sport was taken by the International Amateur Athletic Federation in 1928. The antidoping movement gained real momentum in 1967 when the International Olympic Committee released its first list of prohibited substances. ${ }^{5}$ The anti-doping movement was further facilitated by the formation of the World Anti-Doping Agency (WADA) in 1999 and the subsequent harmonisation of anti-doping policies in most sports and countries. ${ }^{6}$ Testing of sports people for drug use is now regularly conducted both in-competition and out-of-competition for performance-enhancing drugs, including testing for common illicit recreational drugs as a part of in-competition tests.

The use of performance-enhancing drugs has a clear impact on sporting competition. However, the use of illicit drugs, such as cannabis and stimulants, used recreationally, well away from competition time, are less clearly linked to enhanced sporting performance and may even be detrimental. Interestingly, the WADA Code can apply the same sanctions for the use of illicit substances for performance-enhancing effects in competition testing and inadvertent recreational use away from the competition period. ${ }^{5}$ Illicit drugs such as cannabinoids are not widely considered performance enhancing and are presumably on the list under the health risk criteria. ${ }^{7}$ However, the WADA Prohibited List includes cannabinoids in the in-competition testing ${ }^{6}$ but not non-competition testing when the health risks would be the same.

Alongside the health risks of illicit substance use, elite athletes also have a welfare risk because they are subject to sanctions for drug use under their sport's anti-doping codes. Athletes risk sanctions of 2 years or more and for professional athletes such as Australian Football League (AFL) players this has a substantial impact on their welfare.

Because of the differences between substances tested for in-competition and those tested for out-of-competition under the WADA Code, and considering anecdotal reports of drug use and the advice of experts and health promotion organisations, the AFL introduced an Illicit Drugs Policy (IDP) in 2005. This policy started after extensive expert and stakeholder consultation and agreement and the support of the AFL Players Association. The new strategy was introduced to strengthen the AFL Anti-Doping Code (ADC) and close the gap in illicit substance testing by including the non-competition period.

The AFL IDP places player health and welfare as its primary goal. The policy includes League-wide unlocked scheme, see http:// bjsm.bmj.com/site/about/ unlocked.xhtml 
education and the first two detections are responded to with confidential athlete counselling, treatment and rehabilitation. Sanctions occur with the third detection. Since inception, the programme has evolved with increased test numbers, better test targeting and improved medical interventions after detection.

The programme runs in parallel with the WADA compliant AFL ADC that primarily targets performance-enhancing drugs and illicit substances in game day testing. This paper reports the outcomes of the first 7 years of the AFL IDP.

\section{METHODS}

Players from the 16 clubs participated in drug testing under the $\mathrm{ADC}$ and IDP. Each club had a playing list of approximately 40 male players (approximately 640 players), indicative mean age, height and weight of the players in the period 2005-2010 was 23 years, $188 \mathrm{~cm}$ and $87 \mathrm{~kg}$. All players in the AFL were tested under the two drug policies on game days and at any training session during the preseason and competition season. During the players' holiday break (8 weeks) players return to their club once at which time they could be tested under the IDP. Immediately after the holiday break when the players have returned to training in preparation for the AFL competition there is a period of increased IDP testing.

The ADC testing examined urine specimens for both performance-enhancing drugs and recreational drugs (WADA S1-S9 and M1-M3) in competition tests and the IDP examined specimens for commonly used drugs classified under WADA as S6-S8 (cocaine, ecstasy, amphetamines, narcotics and cannabis) as well as $\gamma$-hydroxybutyric acid and ketamine.

The ADC testing was conducted by the Australian Sport Anti-Doping Authority (ASADA) and IDP testing was conducted by a commercial pathology laboratory. Random and targeted tests were conducted. Players were picked for random tests from a list of players at a training session by the testing officials. Targeted tests were directed at players with a previous history of a positive drug test and players who had not been tested in the past 12 months.

In-competition tests were conducted at the playing venue with the drug test personnel notifying the players and collecting the specimens after the game according to WADA protocols. Standard testing privacy and collection procedures were used. Non-competition testing followed similar protocols but were conducted at team training facilities.

Drug testing is part of the players' contract with the AFL and while players did not sign informed consent, the provision of data to third parties for authorised research as overseen by club medical officers is part of the standard playing contract. This manuscript was generated out of the AFL data which is in the public domain. An exemption from ethics was obtained from Monash University, Australia.

\section{RESULTS}

There was a steady decline in the annual number of positive tests (22-6) over the 7 years of the programme. There were six illicit substance detections under the IDP testing programme during 2011 and the detection rate was $0.4 \%$ compared to $4.03 \%$ in 2005 when the programme started. There was a significant reduction in the proportion of positive tests between 2005 and 2011 (table 1, McNemars test, $\chi^{2}=430.4, p<0.0001$ ). The mean age of players who had a first detection was 23.8 years (range $19-30$ years, median 23 years).

The reduction in positive tests has coincided with a $350 \%$ increase in the number of tests conducted and the shift of IDP
Table 1 Results of drug tests for illicit substances

\begin{tabular}{lllll}
\hline Year & $\begin{array}{l}\text { Total test } \\
\text { numbers }\end{array}$ & $\begin{array}{l}\text { Total } \\
\text { detections }\end{array}$ & $\begin{array}{l}\text { Players recording a } \\
\text { second positive* }\end{array}$ & $\begin{array}{l}\text { Detection } \\
\text { (\%) }\end{array}$ \\
\hline 2005 & 472 & 19 & 3 & 4.03 \\
2006 & 486 & 9 & 0 & 1.85 \\
2007 & 1152 & 14 & 3 & 1.20 \\
2008 & 1220 & 12 & 2 & 0.98 \\
2009 & 1568 & 14 & 2 & 0.89 \\
2010 & 1654 & $6 \dagger$ & 1 & 0.36 \\
2011 & 1489 & 6 & 0 & 0.40 \\
\hline
\end{tabular}

*This column reflects those players who recorded their second detection in that year. In 2005, there were 19 detections involving 16 players. Detections 'expire' after 4 years.

†This figure includes a player whose detection was not a positive drug test.

testing times to high-risk periods such as the period immediately after a game when a player was socialising or early in the postseason when players start their break from competition and training.

All detections (80) were from the IDP testing. There were no positive drug tests from the competition tests conducted under the AFL ADC. Aside from the decrease in detections, there has been a trend away from the use of cannabinoids (table 2).

The association with alcohol consumption was compelling; 56 detections were associated with alcohol use, six were not and the data on the remaining 18 was not collected in the first year of the programme. Interestingly, illicit drug use was mostly conducted away from team mates; only six detections were associated with drug use with a team mate, 65 were not and 9 were unknown.

\section{DISCUSSION}

This paper reports on detections of illicit substances in a large group of elite male Australian football players. There has been a steady decline in the number of detections in the 7 years of the IDP in the face of a substantial increase in the number of tests conducted, targeting of players and targeting of high-risk periods. Most detections occurred in the non-competition period, suggesting that illicit substance use was opportunistic, discretionary and not for any performance enhancement effects.

The results of testing over 7 years suggest that the AFL IDP is an effective programme in modifying player behaviour thereby positively impacting on the health and welfare of players. The determination of the AFL and the AFL Players Association in addressing the issue of illicit drug use by players and the commitment of the players themselves and their teams has contributed to the success of the policy.

The IDP additionally appears to have induced behavioural change in players as seen in the reduction in cannabinoid use. Cannabinoids have a long detection window of up to 6 weeks

Table 2 Substances detected with drug testing

\begin{tabular}{llclc}
\hline Year & Cannabinoids & Stimulants & Mixed & Total \\
\hline 2005 & 6 & 12 & 1 & 19 \\
2006 & 0 & 8 & 1 & 9 \\
2007 & 4 & 10 & 0 & 14 \\
2008 & 3 & 8 & 1 & 12 \\
2009 & 1 & 13 & 0 & 14 \\
2010 & 1 & 5 & 0 & 6 \\
2011 & 0 & 6 & 0 & 6 \\
\hline
\end{tabular}


(compared with stimulants of 3-4 days) and out-of-competition testing reduces the opportunity to use cannabis without detection to almost zero as the players only have an 8-week break from playing or training. It appears players have responded to the greater detection risk of cannabinoids.

Most literature reporting on drug use has relied on player survey $^{8} 9$ and very little has been written about the outcomes of drug testing in other sports. Rossi et al ${ }^{10}$ reported the outcomes of 95000 ADC mostly in-competition tests $(15 \%$ of tests were out-of-competition) over 9 years in elite sports participants in Italy. They demonstrated that between 1\% and $1.8 \%$ of tests were positive to all prohibited substances, the incidence fluctuating over the 9 years but not trending down. They did note a high proportion of the positive tests involved stimulants and drugs of abuse and further deduced that their intake appeared not to be for performance enhancement but rather for activities not related to sport.

Illicit recreational drugs comprise a high proportion of the positive tests that arise from competition testing in WADA compliant elite sport. In Australia, WADA compliant testing across all sports is undertaken by ASADA. In a 4-year period the ASADA testing had a detection rate of $0.38 \%$ for all prohibited substances across all sports. Of this total number of ASADA tests the detection of illicit substances at in-competition tests was $0.31 \%$ or over $80 \%$ of the total. ${ }^{2}$ However it is not clear, except in the case of cannabinoids, whether these substances were used by athletes for potential performance-enhancing effects or for recreational purposes and therefore inadvertent from a sport performance perspective.

There is a suggestion that more testing may reduce illicit drug use $e^{5}$ and this study supports this supposition given the decrease in positive tests with greater test numbers. Professional football players in England reported widespread use of recreational drugs in an environment of low testing; only $66 \%$ had been tested in the previous 2 years and $60 \%$ felt they were unlikely to get tested that year. ${ }^{9}$ It would appear that more testing is likely to impact on athlete behaviour.

The operational approach of the AFL IDP includes education across the whole player group and initially confidential clinical management overseen by AFL Medical Officers for players with detections. This approach has evolved over the 7 years of the IDP with the introduction of mandatory club doctor notification and mandatory independent addiction medicine specialist assessment for players with positive tests. In the AFL IDP the results of the first two detections remain confidential between the player and clinicians. A third detection results in referral to the AFL General Manager Football Operations and an open hearing and mandatory suspension of up to 12 games if the player is found guilty. The policy is based on a harm minimisation model rather than a punitive one, ${ }^{11}$ and the primary management approach is focused on prevention and early intervention. The non-adversarial nature of the IDP is highlighted by the fact that 77 of 80 players in this study admitted use after detection (two players had no memory of the event, one player believed their drink had been spiked). The WADA management of illicit substance use is exclusively focused on sanctions without regard to an athlete's intent and risk both to themselves and their sport. ${ }^{11}$ The results of this paper demonstrate that harm minimisation strategies alongside sanctions should be considered in the management of illicit substances. Furthermore, this study demonstrates that in an elite sporting competition, an IDP can effectively impact on athlete use of illicit substances in the out-of-competition period and complement a WADA compliant ADC.

\section{What this study adds}

- Harm minimisation is an effective approach to managing illicit drug abuse alongside existing established punitive methods.

- These data, particularly in the case of cannabinoids, suggest that 'performance enhancement' can be an absolute criterion before a substance is added to the WADA Prohibited List. The inclusion of substances that are primarily harmful to health or in contravention of the spirit of sport can be dealt with by an alternative mechanism.

Acknowledgements The authors wish to acknowledge the assistance of the AFL, the AFL Players Association, AFL Research Board and the AFL Medical Officers Association. JLC was supported by the Australian Centre for Research into Sports Injury and its Prevention, which is one of the International Research Centres for Prevention of Injury and Protection of Athlete Health supported by the International Olympic Committee (IOC).

Contributors $\mathrm{PRH}$ and $\mathrm{HU}$ collected and collated the data in their role with the Australian Football League. JLC reviewed the data, provided statistical and scientific input. All three authors contributed to the writing of the manuscript.

\section{Competing interests None.}

Ethics approval Ethics exemption granted by Monash University.

Provenance and peer review Not commissioned; externally peer reviewed.

Data sharing statement All data in this report are already in the public domain.

\section{REFERENCES}

1. Teesson M, Hall W, Lynskey M, et al. Alcohol- and drug-use disorders in Australia: implications of the National Survey of Mental Health and Wellbeing. Aust N Z J Psychiatry 2000;34:206-13.

2. Australian Institute of Health and Welfare. Statistics on drug use in Australia in 2006.AlHW:Canberra, 2007

3. Dunn M, Thomas J0, Swift W, et al. Recreational substance use among elite Australian athletes. Drug Alcohol Rev 2011;30:63-8.

4. Green GA, Uryasz FD, Petr TA, et al. NCAA study of substance use and abuse habits of college student-athletes. Clin J Sports Med 2001;11:51.

5. Fridman S, Davies C, Amos A. Should athletes be tested for recreational drugs? Three sporting federations kick around the proverbial football. Aust N Z Sports Law J 2007;2:59-77.

6. Australian Sports Anti-Doping Authority. 2009:10 Annual Report. Canberra; 2011.

7. Campos DR, Yonamine M, Moreau RL. Marijuana as doping in sports. Sports Med 2003;33:395-9.

8. Uvacsek M, Nepusz T, Naughton D, et al. Self admitted behavior and perceived use of performance enhancing vs psychoactive drugs among competitive athletes. Scand J Med Sci Sports 2011;21:224-34.

9. Waddington I, Malcolm D, Roderick M, et al. Drug use in English professional football. Br J Sports Med 2005;39:e18.

10. Rossi SS, Botre F, Prevalence of illicit drug use among the Italian athlete population with special attention on drugs of abuse: a 10-year review. J Sports Sci 2011;29:471-6.

11. Smith ACT, Stewart B, Drug policy in sport: hidden assumptions and inherent contradictions. Drug Alcohol Rev 2008;27:123-9. 